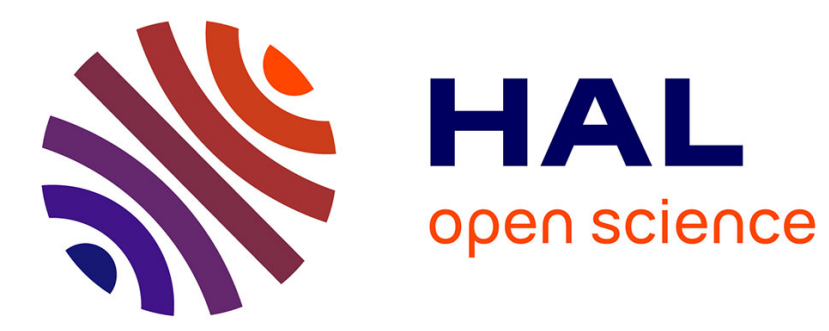

\title{
Hot Spots in an Athermal System
}

Axelle Amon, van Bau Nguyen, Ary Bruand, Jérôme Crassous, Eric Clément

\section{To cite this version:}

Axelle Amon, van Bau Nguyen, Ary Bruand, Jérôme Crassous, Eric Clément. Hot Spots in an Athermal System. Physical Review Letters, 2012, 108, pp.135502-1 - 135502-5. 10.1103/PhysRevLett.108.135502 . insu-00684411

\section{HAL Id: insu-00684411 https://hal-insu.archives-ouvertes.fr/insu-00684411}

Submitted on 2 Apr 2012

HAL is a multi-disciplinary open access archive for the deposit and dissemination of scientific research documents, whether they are published or not. The documents may come from teaching and research institutions in France or abroad, or from public or private research centers.
L'archive ouverte pluridisciplinaire HAL, est destinée au dépôt et à la diffusion de documents scientifiques de niveau recherche, publiés ou non, émanant des établissements d'enseignement et de recherche français ou étrangers, des laboratoires publics ou privés. 


\section{Hot spots in an athermal system}

Axelle Amon ${ }^{1}$, Van Bau Nguyen ${ }^{2,3}$, Ary Bruand ${ }^{3}$, Jérôme Crassous ${ }^{1}$, and Eric Clément ${ }^{2}$

${ }^{1}$ Université de Rennes 1, Institut de Physique de Rennes (UMR UR1-CNRS 6251), Bât. 11A,Campus de Beaulieu, F-35042 Rennes, France ${ }^{2}$ PMMH, ESPCI, UMR CNRS 7636 and Université

Paris 6 \& Paris 7, 75005 Paris, France and

${ }^{3}$ Universit d'Orléans, CNRS/INSU, ISTO UMR 6113, F-45071 Orléans cedex 2, France

(Dated: September 7, 2011) 
Clarification of the mechanisms of plasticity and rupture in amorphous materials is a timely challenge. The state of the art postulates the existence of localized plastic events interacting through long range elastic coupling and triggering irreversible macroscopic deformations [1]. Models assuming such local features at the mesoscopic level are able to reproduce the generic rheology of a large class of materials [2-8]. The activation, dynamics and coupling of such zones have been intensively studied numerically and theoretically [8-14]. However, their concrete existence and their exact role in the various phases of the yield process remain elusive experimentally. Here we visualize directly localized deformation events ('hot spots') in a sheared granular packing using Diffusive Wave Spectroscopy. Their spatial clustering is shown to be linked to the emergence of shear bands. In creep experiments, we relate their rate of apparition to the global plastic deformation rate. We are then able to establish a bridge between our observations and the concept of 'fluidity', recently used to describe nonlocal rheology of several soft materials [15-18].

Our model of amorphous material is an assembly of glass spheres (diameter $200 \pm 30 \mu m$, packing fraction $0.60 \pm 0.01$ ) placed into an axisymmetric cylindrical shear cell (fig 1.a). Shear is obtained by rotation of a four blades vane. The applied stresses and deformations are controlled by a step motor fixed to a torsional spring. Constant motor rotation rate or constant shear stress can be imposed. Two surface loading conditions have been investigated: free and loaded. In addition to the mechanical measurements, we obtain spatially resolved map of the surface deformations using a Diffusive Wave Spectroscopy (DWS) technique [19]. A laser illuminates the top of the shear cell. A camera imaging that surface at a frame rate of $1 \mathrm{~Hz}$, collects backscattered light. The correlation of scattered intensities between two successive images, $g_{I}$, are computed by zone, composing correlation maps of $370 \mu \mathrm{m}$ resolution (fig 1.c). Maximal correlation (light yellow) corresponds to a deformation $\epsilon \lesssim 10^{-7}$ and vanishing correlation (black) corresponds to $\epsilon \gtrsim 10^{-5}$ (see Methods).

We first monitor the mechanical behavior of the system when submitted to an increasing shear stress. Fig 1.b shows the stress as a function of time at a constant motor rotation velocity. The stress increases linearly with the rotation as expected for an elastic material connected to a torsion spring. As the rotation is increased, small stress drops are first observed (precursor events) up to the maximal yield stress value $\sigma_{Y}$. Then, the system 

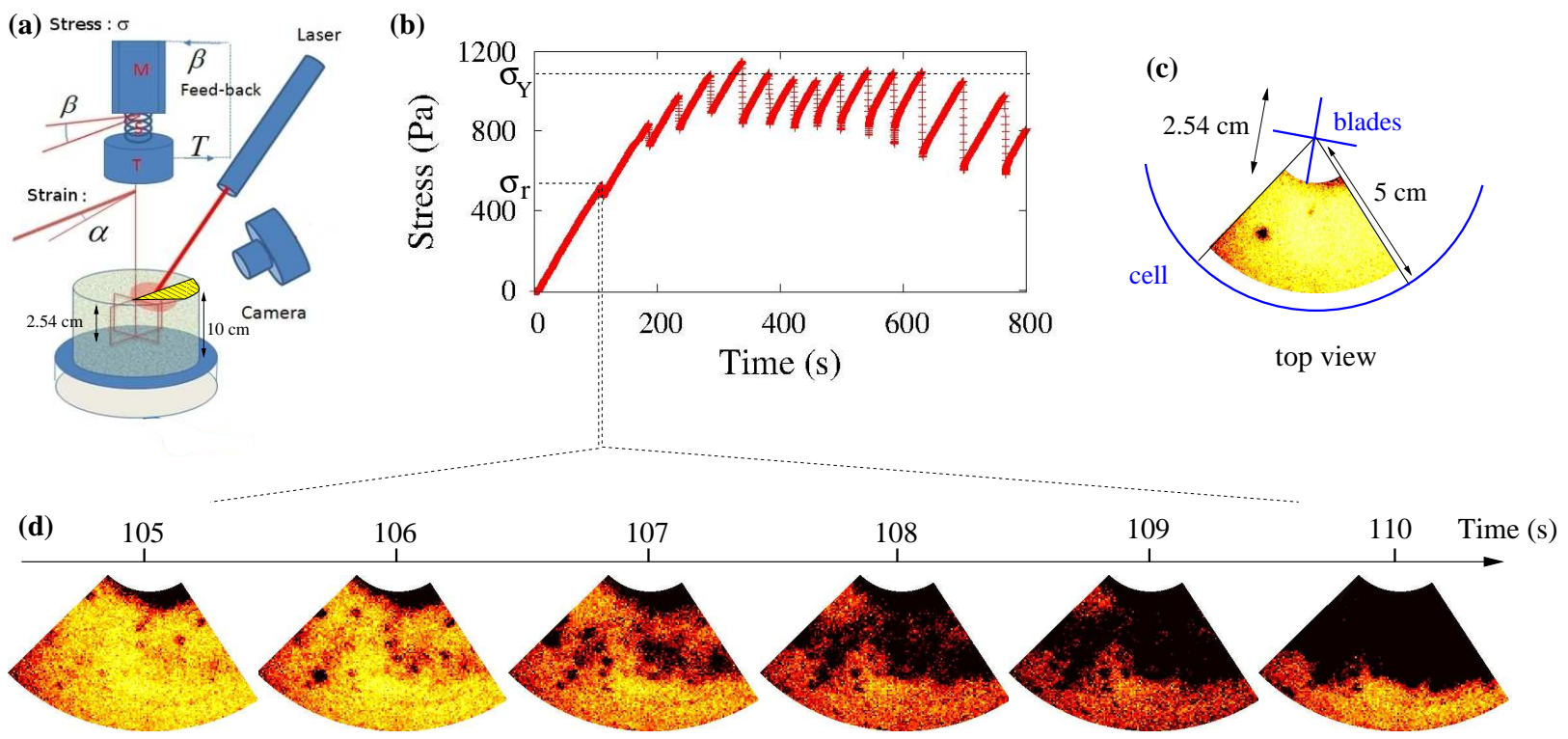

FIG. 1. (a) Schematic of the experimental set-up. (M): motor, (S): Torsion spring, (T): Torque probe. The feed-back loop allows to impose applied torque in creep experiments. A $633 \mathrm{~nm} \mathrm{HeNe}$ Laser beam is expanded and illuminates about a quarter of the top surface. That surface is imaged with a lens on the camera. An iris diaphragm controls the size of the coherence areas on the camera. A polarizer is introduced in the light path to enhance contrast of the speckle pattern. (b) Stress response for an imposed deformation experiment in free surface boundary condition. (c) Part of the surface of the cell that is used for the analysis $\left(S=13 \mathrm{~cm}^{2}\right)$ showing a typical hot spot: in average $g_{I} \simeq 0.99$ (yellow) and $g_{I} \lesssim 0.95$ for the black spot. (d) Correlation maps between consecutive speckle images corresponding to the first stress drop at $\sigma_{r}$. The position of the first failure zone varies from experiments to experiments, and is not systematically situated at the outer edge of the blades as would be observed in Couette cell geometry.

reaches after few rupture events a regular stick-slip motion. This is to be expected for a soft torsion spring, driven at constant rotation rate and coupled with a material displaying both static and dynamic friction thresholds. The onset of precursor events was described by Nguyen et al. [20] and a similar phenomenology was also reported in many other amorphous glassy materials [10]. The DWS imaging of the top surface is shown on fig. 1.c. In the quasielastic part of the loading curves, the intensity correlation stays at $g_{I} \simeq 0.99$ except over small areas, the hot spots, where a significant localized correlation drop down to $g_{I} \lesssim 0.95$ is observed (see fig 1.c and fig 2.a). The extent of these spots is typically $\xi \approx 3 \mathrm{~mm}$ with a 

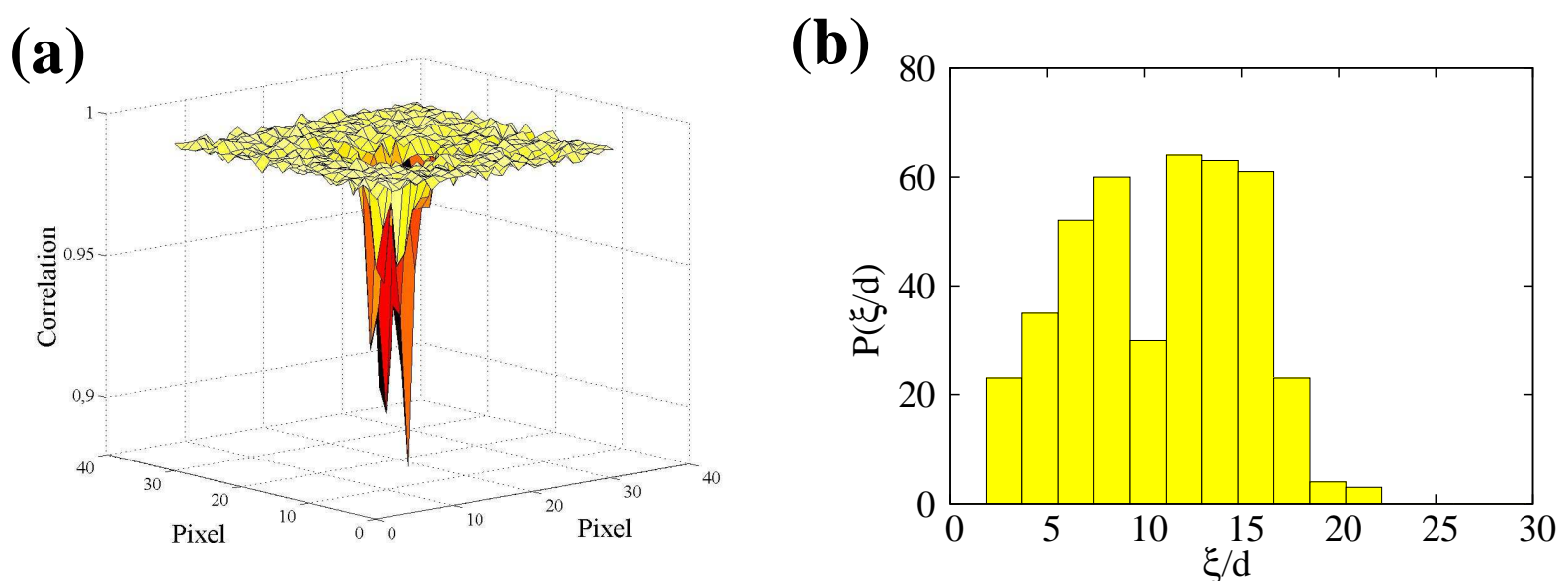

FIG. 2. Characterization of hot spots (a) 3D representation of a typical spot. (b) Distribution of hot spots sizes $\xi$ in units of beads diameter $d$ during a creep experiment.

duration $\tau \sim 1 \mathrm{~s}$. The corresponding local deformation amplitude may be estimated from the decorrelation of the backscattered light [21, 22]: $\epsilon \simeq 5 \times 10^{-6}$ (see Methods). Figure 1.d shows successive maps corresponding to the first stress drop (see also the Supplementary Film). As the deformation is increased, more and more hot spots appear ( $t=105$ to $107 \mathrm{~s})$. These events aggregate in clusters $(t=107$ to $109 \mathrm{~s})$. Finally, a large and totally uncorrelated zone supersedes the clusters $(t=110 \mathrm{~s})$. This large decorrelation zone is associated with the first macroscopic stress drop occurring at stress $\sigma_{r}$. Therefore, the process is two-scales: the localized events occur as precursors of the macroscopic failure inside the glassy material when such failure is itself a precursor event of the final yield.

To link those localized plastic events to the global plastic deformation of the material, we do now creep experiments fixing the applied shear stress and monitoring both the global plastic deformation and the spatially resolved deformation map. On fig. 3 (blue symbols), we display an example of a global plastic deformation $\Delta \gamma(t)$ obtained at low applied shear stress $\sigma=1200 \mathrm{~Pa}$, i.e. at a value smaller than the first precursor event $\sigma_{r}=1700 \mathrm{~Pa}$. During this slow plastic deformation, the DWS imaging shows that the hot spots are still present and appear to be quite isolated at this level of applied stress. To quantify the relation between their apparition and the creep motion, the cumulated number of their occurrence $N(t)$ is computed and displayed on the same graph as $\Delta \gamma(t)$ (fig. 3). The temporal evolution of the two quantities are very similar. Therefore, the plastic deformation rate and the rate of apparition of localized event are proportional. 


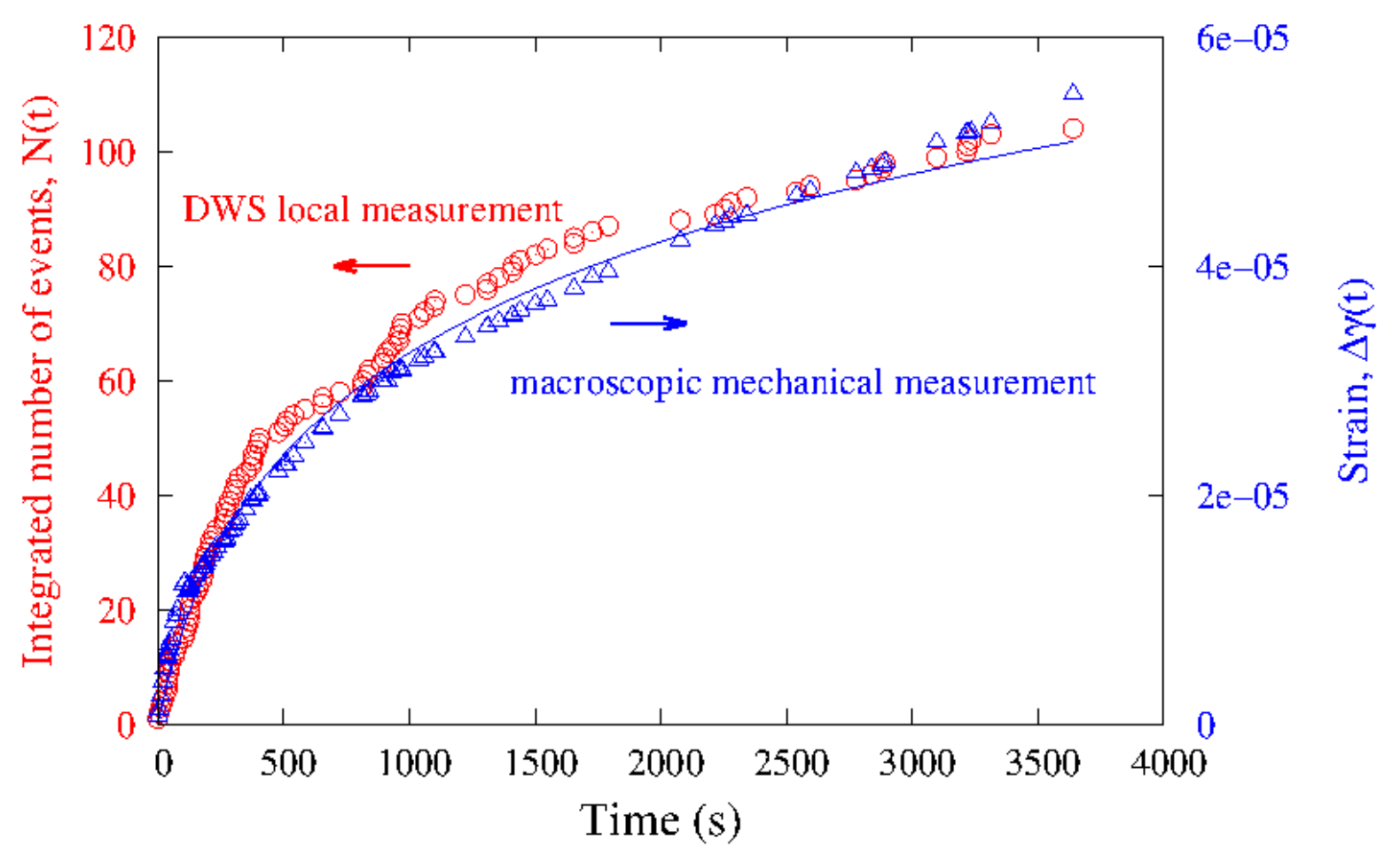

FIG. 3. Blue symbols (right axis): Strain as function of time in a creep experiment at applied shear $\sigma=1200 \mathrm{~Pa}$ for the loaded surface boundary condition. The origin of times is defined as the moment of application of the constant stress, after a linear stress ramp lasting about $100 \mathrm{~s}$. Solid blue line: fit by a logarithmic creep law [20] $\Delta \gamma(t)=\dot{\gamma}_{0} \ln (1+C t) / C$ with $C=0.007 \mathrm{~s}^{-1}$. Red (left axis): integrated number of events.

Such creep experiments can be interpreted using an age dependent relaxation rate [20] reminiscent of many models derived from the 'Soft Glassy Rheology'. In this context a simple visco-elastic scalar equation has been proposed [4] featuring an inverse characteristic relaxation time, the so-called 'fluidity' $f(t)$ :

$$
\dot{\sigma}=G \dot{\gamma}-f(t) \sigma
$$

with $\sigma$ the applied shear stress and $G$ a shear elastic modulus. To close the model, a constitutive relation for $f(t)$ must be provided. Many forms were suggested [4] but for the present case, the observed creep dynamics is consistent with the relation: $\dot{f} \propto-f^{2}$, i.e. a relaxation time proportional to the actual age of the system (see fit of the strain on fig. 3). Building on the proportional relation between the rate of occurrence of hot spots and the shear rate, we propose now to go one step further and to identify $\dot{N}(t)$ with the fluidity 


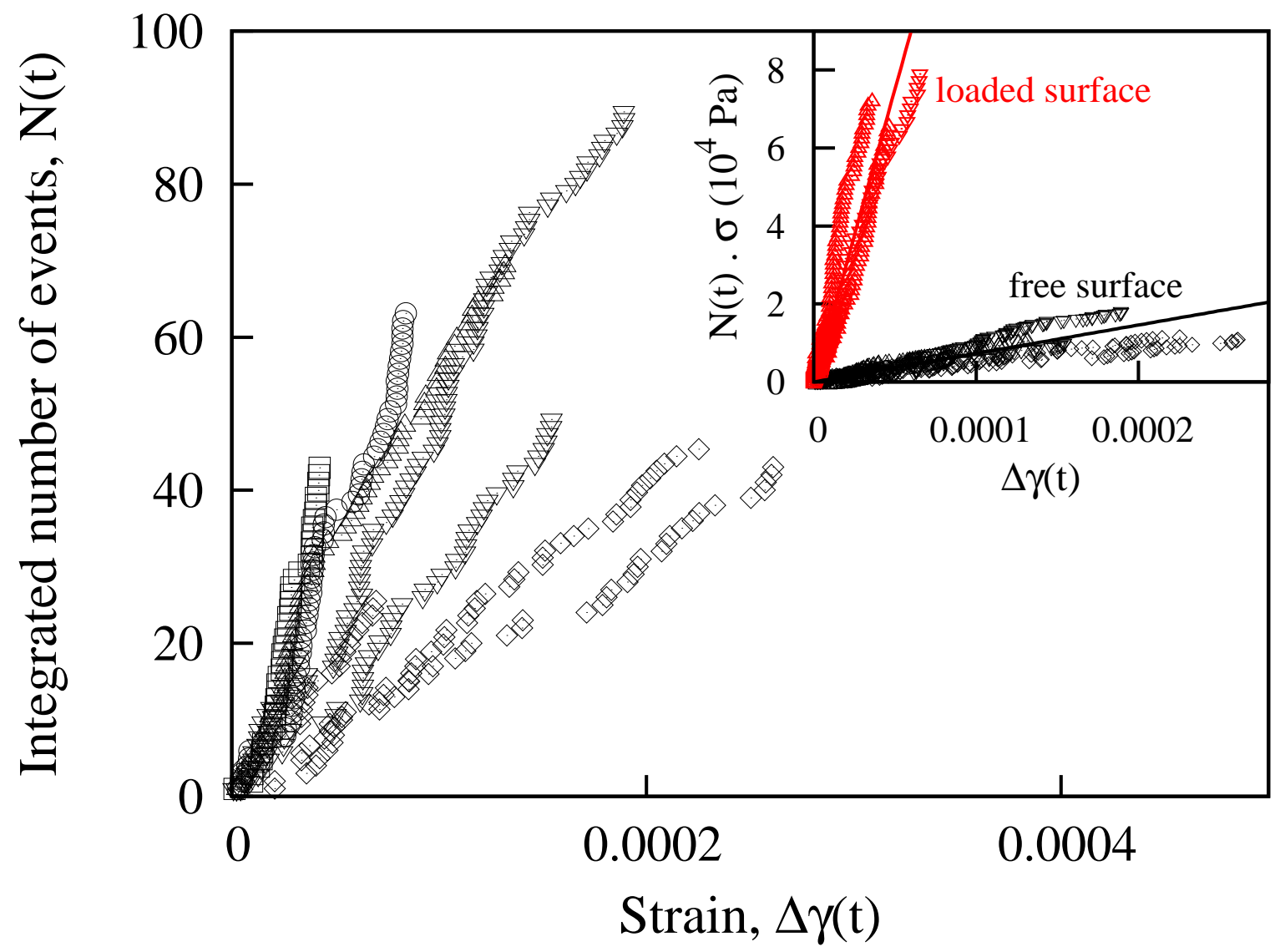

FIG. 4. Integrated number of events in a free surface experiment for different applied stress : 50 $\mathrm{Pa}(\square), 100 \mathrm{~Pa}(\circ), 150 \mathrm{~Pa}(\triangle), 200 \mathrm{~Pa}(\nabla)$ and $250 \mathrm{~Pa}(\diamond)$. Inset : integrated number of events multiply by the apply stress in two configurations. In black, free surface : data from the main graph. In red, loaded surface (top pressure equivalent to $P=830 \mathrm{~Pa}$ ), applied stresses : 400, 800, 1200 and $1600 \mathrm{~Pa}$.

parameter (within a proportionality constant). To test this proposition, we perform series of creep experiments at constant applied shear stresses $\sigma$ for stress values below $\sigma_{r}$. Fig. 4 displays the relation between the cumulated number of events $N(t)$ and the total plastic strain $\Delta \gamma(t)$, for free surface loading condition. The relation is roughly linear, with slopes decreasing with the amplitude of the applied stress. Using our hypothesis of proportionality between the fluidity parameter and the hot spots rate of occurrence, integration of eq.(1) at constant stress gives:

$$
G \Delta \gamma(t)=\sigma \int_{0}^{t} f\left(t^{\prime}\right) d t^{\prime} \propto \sigma N(t)
$$

Following this relation it is then possible to collapse all the curves on a master straight line 
when multiplying $N(t)$ by the applied stress $\sigma$ (inset of fig.4). To evaluate the robustness of this relation, we varied the top surface boundary condition: due to Hertz elasticity, the effective shear modulus $G$ should increase with the confining pressure. Indeed, when the surface is loaded, the linear relation between $N(t) \sigma$ and $\Delta \gamma(t)$ hold (see inset of fig.4) showing the expected qualitative stiffening (a factor 20 approximatively). However from a simple account of the Hertz law, we should only expect here a factor 2. This significant difference is possibly due to the presence near the free surface of a strong stiffness gradient and a diverging elastic susceptibility.

So far, we have established a direct relation between the rate of plastic deformation and the apparition of the hot spots. Their clustering in the vicinity of major yield events prior to their macroscopic apparition, reinforces the idea that they play a leading role in the plasticity process. However, it is not a priori obvious that these are the only and sufficient modes of rupture which account for the full plastic flow dynamics. Therefore, we compare an estimation of the energy released by the hot spots to the total elastic energy dissipated in the bulk. The energy dissipation of one plastic event per unit of depth is $\sigma \epsilon \xi^{2}$, where $\sigma$ is the local stress and $\epsilon \approx \sigma / G$ the typical plastic deformation during one event. We suppose that the 'hot spots' density is uniform in the bulk. During a creep experiment, the density of energy release through the elastic bulk relaxation processes is $\sigma \Delta \gamma(t)$. Then, the energy balance per unit depth, between the sum of the localized plastic dissipation $\sigma \epsilon \xi^{2} N(t)$ and the bulk elastic relaxation $S \sigma \Delta \gamma(t)$ leads to the linear relation: $\sigma N(t)=K \Delta \gamma(t)$ with $K=G S / \xi^{2}$. Quantitatively, the relation can be tested on the surface loaded experiment for which the elastic response $G=1.6 \times 10^{6} \mathrm{~Pa}$ has been measured (our torque probe was not sensitive enough to access to the elastic regime of the free surface packing). We obtain : $K \approx 10^{9} \mathrm{~Pa}$ close to the value obtain on the inset of fig.4. Therefore, within the consistency of the model, it seems that the energy released by the hot spots comes to the right magnitude to account for the macroscopic plasticity.

In this letter, we provide a direct visualization of localized deformation events associated with the mechanical response of a granular packing under shear. The optical DWS technique captures the emergence of the so-called 'hot-spots', characterized by a spatial extension of about ten grains. Under a stress ramp, we show that these events increase in density with the stress amplitude and cluster spatially as precursors of the shear banding macroscopic yield events. Under constant applied shear stress, the rate of apparition of these hot spots 
at the top surface is proportional to the rate of plastic deformation. Varying the applied shear and the loading boundary conditions, we point that at first approximation - possibly corresponding to a mean-field approximate of the problem - the 'hot spot' dynamics is in direct relation with a parameter called fluidity defined in the context of 'Soft Glassy Rheology', and representing the mean rate of stress relaxation. Our study points out the interest of clarifying this relation, by characterizing the spatio-temporal coupling of the hot spot dynamics, in particular in the vicinity of the material yield or under sustained mechanical noise. These results provide some substantial experimental evidences backing many theoretical propositions made recently about zero temperature plasticity of amorphous solids. It focuses the debate on the importance of localized precursor events participating in the yield dynamics, a concept which can be crucial in a wide context encompassing solid disordered system, complex fluids or even earth-quake dynamics.

[1] Argon, A.S. Plastic deformation in metallic glasses. Acta Metallurgica 27, 47-58 (1979).

[2] Sollich, P., Lequeux, F., Hébraud, P. \& Cates M.E. Rheology of soft glassy materials. Phys. Rev. Lett. 78, 2020-2023 (1997).

[3] Sollich, P. Rheological constitutive equation for a model of soft glassy materials. Phys. Rev. E 58, 738-759 (1998).

[4] Derec, C., Ajdari, A. \& Lequeux, F. Rheology and aging: A simple approach. Eur. Phys. J. E 4, 355-361 (2001).

[5] Kabla, A. \& Debrégeas, G. Local stress relaxation and shear banding in a dry foam under shear. Phys. Rev. Lett. 90, 258303 (2003).

[6] Goyon, J., Colin, A., \& Bocquet, L. How does a soft glassy material flow: finite size effects, non local rheology, and flow cooperativity. Soft Matter 6, 2668-2678 (2010).

[7] Dahmen, K.A., Ben-Zion, Y. \& Uhl, J.T. A simple analytic theory for the statistics of avalanches in sheared granular materials. Nature Physics 7, 554-557 (2011).

[8] Lemaître, A. \& Caroli, C. Rate-Dependent Avalanche Size in Athermally Sheared Amorphous Solids. Phys. Rev. Lett. 103, 065501 (2009).

[9] Falk, M.L. \& Langer, J.S Dynamics of viscoplastic deformation in amorphous solids Phys. Rev. E 57, 7192-7205 (1998). 
[10] Tanguy, A., Leonforte, F. \& Barrat, J.-L. Plastic response of a 2d Lennard-Jones amorphous solid: Detailed analysis of the local rearrangements at very slow strain rate. Eur. Phys. J. E 20, 355-364 (2006).

[11] Maloney, C.E. \& Lemaître, A. Amorphous systems in athermal, quasistatic shear. Phys. Rev. E 74, 016118 (2006).

[12] Lerner, E. \& Procaccia, I. Scaling theory for steady-state plastic flows in amorphous solids. Phys. Rev. E 80, 026128 (2009).

[13] Karmakar, S., Lerner, E. \& Procaccia, I. Predicting plastic flow events in athermal shearstrained amorphous solids. Phys. Rev. E 82, 055103(R) (2010).

[14] Talamali, S.M., Petäjä, V., Vandembroucq D., \& Roux, S. Strain localization and anisotropic correlations in a mesoscopic model of amorphous plasticity. Preprint: arXiv:1005.2463 (2011).

[15] Picard, G., Ajdari, A., Bocquet, L. \& Lequeux, F. Simple model for heterogeneous flows of yield stress fluids. Phys. Rev. E 66, 051501 (2002).

[16] Bocquet, L., Colin, A. \& Ajdari, A. Kinetic theory of plastic flow in soft glassy materials. Phys. Rev. Lett. 103, 036001 (2009).

[17] Goyon, J., Colin, A., Ovarlez, G., Ajdari, A. \& Bocquet, L. Spatial cooperativity in soft glassy flows. Nature 454, 84-87 (2008).

[18] Katgert, G., Tighe, B.P., Mobius, M.E. \& van Hecke, M. Couette Flow of Two-Dimensional Foams. Europhys. Lett. 90, 54002 (2010).

[19] Erpelding, M., Amon, A. \& Crassous, J. Diffusive wave spectroscopy applied to the spatially resolved deformation of a solid. Phys. Rev. E 78, 046104 (2008).

[20] Nguyen, V.B., Darnige, T., Bruand, A. \& Clément, E. Creep and fluidity of a real granular packing near jamming. Phys. Rev. Lett. in Press (2011).

[21] Crassous, J. Diffusive wave spectroscopy of a random close packing of spheres. Eur. Phys. J. E 23, 145-152 (2007).

[22] Erpelding, M., Amon, A. \& Crassous, J. Mechanical response of granular media: New insights from diffusing-wave spectroscopy. Europhys. Lett. 91, 18002 (2010).

\section{Acknowledgements}

We acknowledge the financial supports CNRS-PEPS program, the ANR projects "Jamvibe2010" and "STABINGRAM" No. 2010-BLAN-0927-01. E.C. thanks Bruno Andreotti for 
many scientific discussions; A.A. and J.C. thank Patrick Chasles for help with the image acquisition.

\section{Author Contributions}

V.B.N., A.B. and E.C. designed the creep experiment, V.B.N. made creep experiment, A.A. and J.C. designed and made DWS experiments, A.A. did the data analysis and E.C., A.A. and J.C. wrote the paper.

\section{Author Information}

Correspondence and requests for materials should be addressed to E.C. (eric.clement@upmc.fr) or J.C. (jerome.crassous@univ-rennes1.fr)

\section{Methods}

The filling height of the shear cell is $\simeq 10 \mathrm{~cm}$. The penetration depth of the vane is fixed so that the upper edge reaches the surface from below within a few grain sizes. During the insertion procedure the material is gently fluidized by an air flux in order to obtain a controlled uniform compacity, deduced from in-situ permeability measurement (see [20] for details). The fluidization process is stopped during each mechanical measurements. The spring deformation and the rotation of the vane are measured, giving access to the applied stresses and strains. The device is suited to work at an imposed motor rotation rate or at an imposed shear stress due to a feed back loop between the torque probe and the motor. For the loaded surface condition a circular glass lid with a hole for the axis is placed on the glassy material and supplementary loads are added, producing a top pressure equivalent to $P=830 \mathrm{~Pa}$. The backscattering DWS technique probes the deformation field in a plane perpendicular to the shear vorticity axis in a depth corresponding to the light transport mean free path $l^{*}$. The correlation between the successive speckle patterns are computed and averaged over zones of $16 \times 16$ pixels, corresponding to $370 \mu \mathrm{m} \times 370 \mu \mathrm{m}$ areas. Previous work on backscattering DWS technique in granular packing [21, 22] have established a relation $\epsilon \approx-\ln \left(g_{I}\right) / c$ were $c \simeq 15000$ is a numerical constant related to the ratio between $l^{*} \approx 3.3 d$ and the optical wavelength. With $g_{I} \approx 0.95$, we obtain here approximatively $\epsilon \simeq 5 \times 10^{-6}$. 